\title{
Proposta de redimensionamento do plano básico da faixa de TV em função de estudos de casos de exposição populacional a campos elétricos, magnéticos e eletromagnéticos.
}

\author{
Carnot L. B. Guimarães, Thyago O. B. Guimarães, João C. W. A. Costa, e Gervásio P. S. Cavalcante \\ Eletromagnetismo Aplicado, Belém, Pará, carnot@ anatel.gov.br, Brasil
}

\begin{abstract}
Resumo - 0 estudo é baseado em simulações utilizando 0 banco de dados da ANATEL e medidas realizadas em ambientes prediais localizados as proximidades, entre 20 e 120 metros, de estações transmissoras do serviço de TV, caracterizando região de campo distante, nele procura-se verificar os níveis de exposição à radiação não-ionizante (RNI), a que a população em geral, os quais são moradores dos edifícios, estão expostos, tornando-se necessário o imediato redimensionamento das potências irradiadas ou re-alocação das estações de entidades que visam somente à cobertura cada vez maior por um só sistema irradiante.
\end{abstract}

Palavras-chave - Radiação não-ionizante, Estações de TV, População em geral, Níveis de exposição, C ampo distante.

Abstract - The study it is based on simulation having used the data base of the ANATEL and measures carried through in land environments located the neighborhoods, between 20 and $\mathbf{1 2 0}$ meters, of transmission stations of the TV service, characterizing region of distant field, in it is looked to verify the levels of exposition to the non-ionizing radiation (NIR), the one that the population in general, which is living of the buildings, is displayed, becoming necessary the immediate redimension of the radiated powers or reverse speed-allocation of the stations of entities that only aim at to the covering each time greater for only a radiant system.

K eywords - Non-ionizing radiation, TV stations, Population in general, L evels of exposition, Distant field.

\section{INTRODUÇÃO}

Há muito tempo se discute sobre os prováveis efeitos da radiação não-ionizante nos seres humanos, tendo como principal fonte às estações transmissoras de radiocomunicação de serviços de telecomunicações. Vários países desenvolveram pesquisas e criaram legislação própria relacionada a este assunto.

A divisão de saúde ambiental da Organização Mundial de Saúde (OMS) e a IRPA/INIRC desenvolveram juntas vários documentos, os quais fazem parte do programa de critérios de saúde ambiental da OMS, patrocinado pelo Programa Ambiental das Nações Unidas (United Nations Envairomental Programme - UNEP). Cada documento inclui uma visão geral das características físicas, técnicas de medição e instrumentação, fontes e aplicações de RNI. Estes critérios proporcionaram uma base de dados científica para posterior desenvolvimento dos limites de exposição e dos procedimentos relacionados a RNI.

No ano de 1992, em substituição a IRPA/INIRC, foi criada uma nova organização científica internacional independente, a Comissão Internacional de Proteção contra as Radiações Não-Ionizantes (International Commission or Non-Ionizing Radiation Protection - ICNIRP), a qual tem como função investigar os perigos que podem estar associados com as diferentes formas de RNI, desenvolver diretrizes internacionais sobre os limites de exposição a RNI e também tratar de todos os aspectos de proteção a RNI.

Em 1998, a ICNIRP anunciou diretrizes que recomendam os limites de exposição à radiofreqüência, fornecendo grandes perspectivas de proteção para a população mundial. Essas diretrizes vêm sendo amplamente adotadas em muitos países, se tornando padrões de segurança mundial.

No Brasil, a resolução no 303/2002 da Agência Nacional de Telecomunicações - ANATEL, aderiu às normas do ICNIRP e aprovou o Regulamento sobre Limitação da Exposição a Campos Elétricos, Magnéticos e Eletromagnéticos na faixa de radiofreqüência entre $9 \mathrm{KHz}$ e $300 \mathrm{GHz}$.

Baseando-se nos métodos de medição definidos na própria resolução foram realizadas medidas em 4 sites localizados na área metropolitana da cidade de Belém-Pá, onde se concluiu pela necessidade do remodelamento do modelo de plano básico dos Serviços de Transmissão de TV hoje implantados no Brasil, de forma a diminuir as potências EIRP radiadas por estas estações transmissoras de alta potencia ou retirá-las de dentro dos grandes centros urbanos.

Este artigo visa mostrar os altos níveis a que estão expostos os cidadãos comuns na região metropolitana de uma grande capital brasileira.

\section{INTERAÇÃO DA RADIAÇÃO NÃO-IONIZANTE COM A MATÉRIA}

Nesta seção serão apresentados alguns conceitos da interação dos campos elétricos, magnéticos e eletromagnéticos com a matéria, onde os mesmos são provenientes de fontes de radiação não-ionizante. Faz-se necessária esta apresentação, para mostrar que a faixa de radiofreqüência de transmissão de sons e imagens, a qual é objeto de estudo do presente artigo, é a que mais traz problemas ao ser humano.

Para que a radiação eletromagnética possa produzir algum efeito, em um tecido ou em qualquer outra substância é necessário que haja transferência de energia desta radiação para o meio, e que esta energia seja absorvida. Os efeitos desta absorção no tecido humano são 
de naturezas térmicas ou não térmicas, dependendo se os efeitos são devidos à decomposição de calor ou devido à interação direta do campo com as substâncias, sem transferência significativa de calor. Os fatores mais importantes, para a absorção das ondas são: constante dielétrica, condutividade, geometria, e conteúdo de água do meio [1].

\subsection{Materiais Dielétricos}

De acordo com a equação de Poisson, a fonte do deslocamento dielétrico D é dada pela densidade de cargas livres (conduzindo) ñ:

$$
\operatorname{div} \mathbf{D}=\tilde{n ̃}_{\text {livre }}
$$

A neutralidade total de carga da matéria em um campo externo é descrita por [1]:

$$
\mathbf{D}=\varepsilon_{0} \mathbf{E}+\mathbf{P}
$$

A contribuição do vácuo causada pelo campo elétrico externamente aplicado é representada pelo termo $\mathcal{E} \mathbf{0}$ E, e a polarização elétrica da matéria no sistema é descrita pelo $\mathbf{P}$ [1]. Esta relação é independente da natureza da polarização. Para uma resposta dielétrica pura da matéria a polarização é proporcional ao campo elétrico em uma aproximação linear por:

$$
\mathrm{P}=\varepsilon_{0} \mathrm{X} \text { eE ou } \mathrm{D}=\varepsilon_{0} \varepsilon r E
$$

A susceptibilidade do dielétrico $X$ é relacionado à constante dielétrica relativa $\varepsilon r$ pelo $X=\varepsilon r$ 1. Além disso, a resposta dielétrica depende dos campos diagonais. As equações (2) e (3) descrevem as propriedades médias do dielétrico. Este ponto de vista macroscópico não considera a origem microscópica da polarização [2]. A polarização macroscópica $\mathrm{P}$ é a soma de todo o pj individual dos momentos de dipolo do material com a densidade $\mathrm{Nj}$.

$$
P=\sum \text { Njpj }
$$

Uma equação similar descreve a relação entre a indução magnética $\mathrm{B}$ e o campo magnético externo $\mathrm{H}$, em um meio isotrópico.

$$
\mathrm{B}=\mathrm{K}_{\mathrm{m}} \mu_{0} \mathrm{H}
$$

onde $\mathrm{K}_{\mathrm{m}} \mathrm{e}^{\mu_{0}}$ são a permeabilidade magnética do material e a permissividade do vácuo, respectivamente..

\subsection{Constante Dielétrica dos Tecidos}

Os valores das constantes dielétricas de diferentes tecidos dependem da constituição dos mesmos, da freqüência e em caso de moléculas polares, também da temperatura. Em relação à água, que é uma molécula polar, a constante dielétrica relativa é 81 para baixas freqüências, devido à inércia rotacional dos dipolos elétricos com o campo externo. Nos tecidos gordurosos, a constante dielétrica é baixa, assim por exemplo a $900 \mathrm{MHz}$, um tecido adiposo com $10 \%$ de água possui $\varepsilon_{\mathrm{r}}=4$ enquanto com $50 \%$ de água o mesmo tecido possui $\varepsilon_{\mathrm{r}}=12$. Devido a esta variação com a concentração de água é difícil prever o comportamento dielétrico dos tecidos em experiências de laboratório. A dependência com a temperatura é da ordem de $2 \% /{ }^{\circ} \mathrm{C}$ [1].

\subsection{Condutividade Específica dos Tecidos}

A condutividade dos tecidos varia de forma significativa com a frequiência para valores acima de $1 \mathrm{GHz}$, como se observa na Fig. 1, para o sangue. Este gráfico mostra o comportamento de tecidos com alto grau de água.

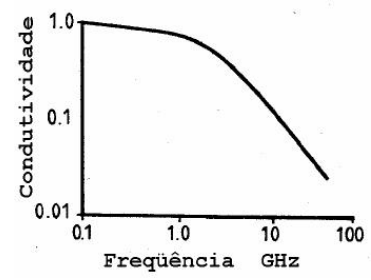

Fig. 1. Condutividade específica do sangue em função da frequiência [2].

A potência absorvida por unidade de volume, $\mathrm{P}_{\mathrm{a}}$, por uma onda incidente com o campo elétrico $\mathrm{E}$ e um tecido de condutividade $\sigma$, é dada por:

$P_{a}=\frac{\sigma|E|^{2}}{2}$

\subsection{Profundidade de Penetração}

Entende-se por Efeito Skin ou Efeito Pelicular, como sendo a profundidade em uma substância na qual a amplitude da radiação é reduzida em 1/e (37\%) do valor incidente, e a densidade de potência, em $1 / \mathrm{e}^{2}$ ou seja a $13,5 \%$, portanto $86,5 \%$ da energia é dissipada na película de espessura $\delta[1]$.

A relação entre a profundidade de penetração $\delta$, com a frequiência é dada por:

$\delta=\left(\frac{\rho}{\pi f \mu}\right)^{\frac{1}{2}}$

onde $\rho$ é a resistividade [ $[\Omega m]$ e $\mu$ é a permeabilidade magnética do tecido.

\subsection{Taxa de Absorção Específica}

Uma das grandezas físicas de maior interesse na quantificação de limites básicos de exposição às radiações eletromagnéticas é a Specific Absoption Rate (SAR) - Taxa de Absorção Específica. Essa grandeza representa a taxa de potência absorvida por unidade de massa e é dada em watt por quilo $[\mathrm{W} / \mathrm{kg}]$ e é usada em medidas ou cálculos de corpopresente. Ela representa a média espacial sobre toda a massa exposta a radiações de frequiências maiores que 10 $\mathrm{MHz}$, porque para freqüências menores o conceito de SAR perde o significado, visto que os efeitos biológicos resultantes da exposição humana, são melhores correlacionadas com as densidades de corrente resultantes 
no corpo. A SAR é também considerada como sendo a variação no tempo do aumento da energia absorvida, dW num elemento de volume $\mathrm{dV}$ de massa $\mathrm{dm}$, e densidade ${ }^{\rho}$, e é dado por [1]:

$$
\begin{aligned}
& \text { SAR }=\frac{d}{d t}\left(\frac{d W}{d m}\right)=\frac{d}{d t}\left(\frac{d W}{\rho d V}\right) \\
& S A R=\left(\frac{\sigma}{2 \rho}\right)|E|^{2}
\end{aligned}
$$

onde $\sigma$ é a condutividade da massa do corpo onde é absorvida a radiação.

Observa-se que a SAR é diretamente proporcional ao aumento local de temperatura, responsável pelos efeitos térmicos, ou seja:

$$
\frac{\mathrm{dT}}{\mathrm{dt}}=\left(\frac{1}{\mathrm{C}_{\mathrm{p}}}\right) \mathrm{SAR}
$$

onde $\mathrm{T}$ é a temperatura e $\mathrm{C}_{\mathrm{p}}$ é o calor específico do tecido $\left[\mathrm{J} / \mathrm{kg} .{ }^{\circ} \mathrm{C}\right]$.

Para exposição do corpo inteiro, pode-se considerar a SAR média, porque em diferentes locais como mãos, punhos, tornozelos e pés este valor de SAR é diferente, a SAR será a relação entre a potência total absorvida pelo corpo e sua massa.

A dependência da freqüência da SAR pode ser dividida em três partes. Na região de mais baixa frequiência, abaixo de $30 \mathrm{MHz}$, a energia de absorção diminui rapidamente com a diminuição da freqüência. Os efeitos não térmicos são predominantes na região principalmente de freqüências muito baixas $(<300 \mathrm{kHz})$.

Na região de ressonância, entre (30 MHz e $400 \mathrm{MHz})$, o tamanho do corpo e o comprimento de onda são da mesma ordem de grandeza e por isso a absorção da radiação é maior e os efeitos térmicos predominam, faixa esta que inclui o serviço de transmissão de sons e imagens objeto de estudo do presente artigo.

Nas regiões de maiores freqüências, (> $400 \mathrm{MHz}$ ), $\lambda$ é menor, a penetração da radiação é menor, e pode ocorrer a produção de locais sobre-aquecidos em regiões do corpo, como por exemplo, na cabeça.

\section{NORMAS E LIMITES DE EXPOSIÇÃO À RADIAÇÃO NÃO-IONIZANTE}

As normas de exposição são elaboradas para proteger a população em geral contra os danos da radiação não ionizante, proveniente da radiofreqüência. O público em geral consiste de pessoas de todas as idades e estados de saúde e pode incluir grupos ou indivíduos particularmente suscetíveis, em muitos casos, essas pessoas não têm consciência de sua exposição aos campos eletromagnéticos (CEM). É sobre esta consideração que se baseia a adoção de restrições mais rigorosas para a exposição á este tipo de radiação.
As restrições aplicáveis a efeitos biológicos da exposição a CEM baseiam-se em efeitos bem fundamentados sobre a saúde e são denominadas restrições básicas. Dependendo da frequiência, as grandezas físicas usadas para especificar as restrições básicas sobre a exposição a CEM variam, podem ser a densidade de corrente, a SAR ou a densidade de potência. A proteção contra efeitos prejudiciais à saúde requer que estas restrições básicas não sejam excedidas.

Níveis de referência de exposição são fornecidos para comparação com valores medidos de grandezas físicas. A concordância com todos os níveis de referência, garante a concordância com as restrições básicas. No caso dos valores medidos serem maiores do que os níveis de referência, isso não significa necessariamente que as restrições tenham sido excedidas, mas uma análise mais detalhada torna-se necessária para avaliar a concordância com as restrições básicas.

No Brasil, a primeira proposta, a nível nacional, de normatizar a exposição a RNI surgiu em julho de 1999, com a decisão da ANATEL de adotar, como referência provisória, os mesmo limites propostos pela ICNIRP. O documento foi então traduzido para o português pela Associação Brasileira de Compatibilidade Eletromagnética (ABRICEM). Em 02 de julho de 2002 entrou em vigor a Resolução No 303 da ANATEL com o "Regulamento sobre Limitação da Exposição a Campos Elétricos, Magnéticos e Eletromagnéticos na Faixa de Radiofrequiências entre $9 \mathrm{kHz}$ e 300 GHz", que estabelece, além dos limites à exposição à RNI, métodos de avaliação e procedimentos para licenciamento das estações de telecomunicação. O conteúdo deste regulamento tem bases nas diretrizes da ICNIRP e nas contribuições recebidas em decorrência de duas consultas públicas realizadas em abril e maio de 2001. Os limites não sofreram alteração em relação aos valores determinados pela ICNIRP [3].

A Fig. 2 ilustra os limites de campo elétrico $(\mathrm{V} / \mathrm{m})$ e de densidade de potência $\left(\mathrm{W} / \mathrm{m}^{2}\right)$, para exposição ocupacional e da população em geral, em conformidade com a resolução 303/2002 da ANATEL.

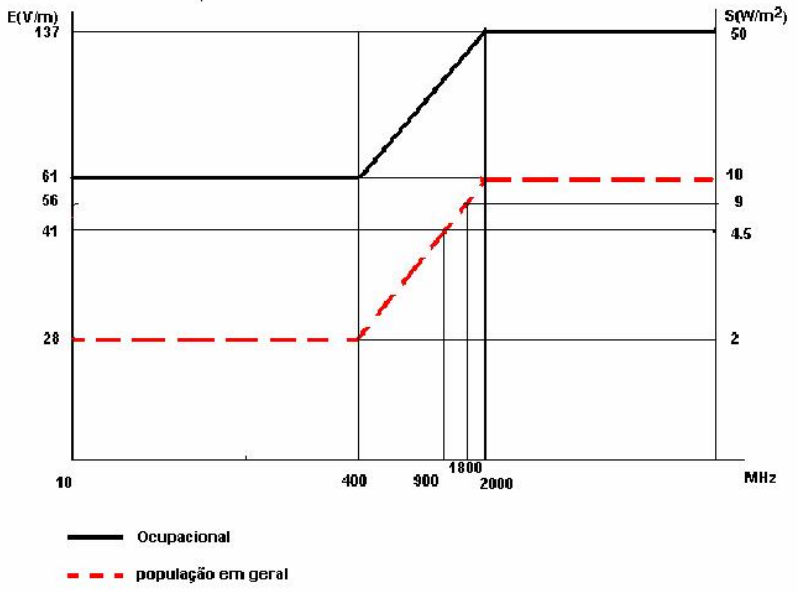

Fig. 2. Limites de exposição ao campo elétrico e densidade de potência em função da freqüência de acordo com a Resolução 303/2002 da ANATEL [3]. 
Nas medições em questão, o instrumento utilizado em campo foi assim constituído:

Para medição seletiva em freqüência:

- $\quad$ Analisador de Espectro Portátil FSH-3 e Sonda Isotrópica, de fabricação Rohde \& Schwarz. Especificações no site do fabricante.

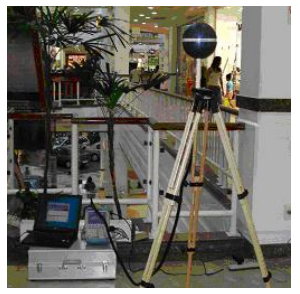

Fig. 3. Foto da sonda e do Analisador de Espectro

\section{CÁLCULOS TEÓRICOS E MEDIDAS INDOOR DOS CAMPOS ELÉTRICOS}

Nesta seção serão apresentados os cálculos teóricos e os valores medidos com a sonda e o analisador de espectro.

Os pontos de referência para os cálculos e medições serão mostrados a seguir:

\subsection{TV SBT Canal 5 de Belém S/A}

Latitude: 01s27' 32,0”.

Longitude: 48s28'41,0".

Antena: situada a 110.25 metros

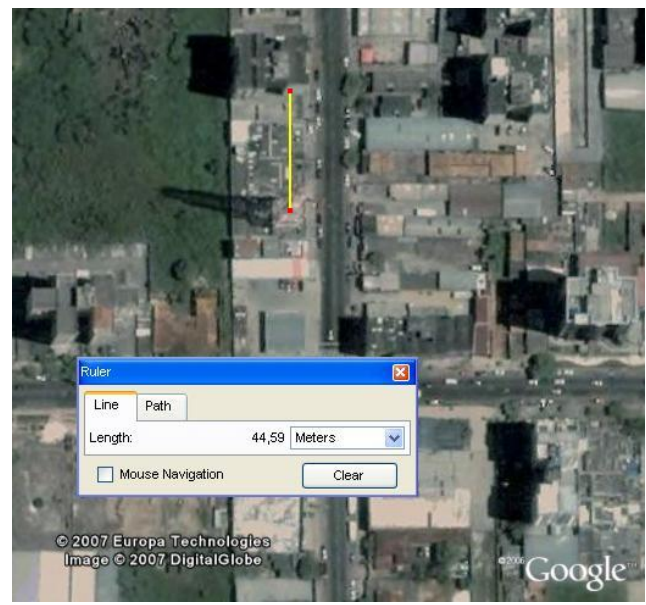

Fig. 4. Foto da antena do SBT e o ponto de medição.

\section{TABELA 1}

Cálculo e Medição do campo elétrico do sistema irradiante de radiodifusão de sons e imagens da TV SBT

\begin{tabular}{|c|c|}
\hline PARÁMETROS & 1 \\
\hline Potência Máxima do Transmissor $\{\mathrm{W}\}$ & 20000 \\
\hline Perdas totais no Sistema Lsistema $\{\mathrm{dB}\}$ & 2,40 \\
\hline Perdas Totais no Sistema Isistema $\{$ Linear $\}$ & 1,74 \\
\hline Ganho da Antena Gantena $\{\mathrm{dBi}\}$ & 8,02 \\
\hline Ganho da Antena Gantena $\{$ linear $\}$ & 6,34 \\
\hline $\operatorname{eirp}\{\mathrm{W}\}=$ Pxmt $x$ Gantena / Isistema & 72951 \\
\hline DENSIDADE DE POTÊNCIA $\left\{\mathrm{W} / \mathrm{m}^{2}\right\}$ & 7,475 \\
\hline CAMPO MAGNÉTICO $\{\mathrm{A} / \mathrm{m}\}$ & 0,141 \\
\hline $\begin{array}{l}\text { CAMPO ELÉTRICO CALCULADO }\{\mathrm{V} / \mathrm{m}\} \\
\text { CAMPO ELÉTRICO MEDIDO }\{\mathrm{V} / \mathrm{m}\}\end{array}$ & $\begin{array}{l}53,084 \\
47,772\end{array}$ \\
\hline
\end{tabular}

\subsection{Televisão Liberal LTDA}

Latitude: 01s25'33,0".

Longitude: 48s30'00,0".

Antena: situada a 113 metros

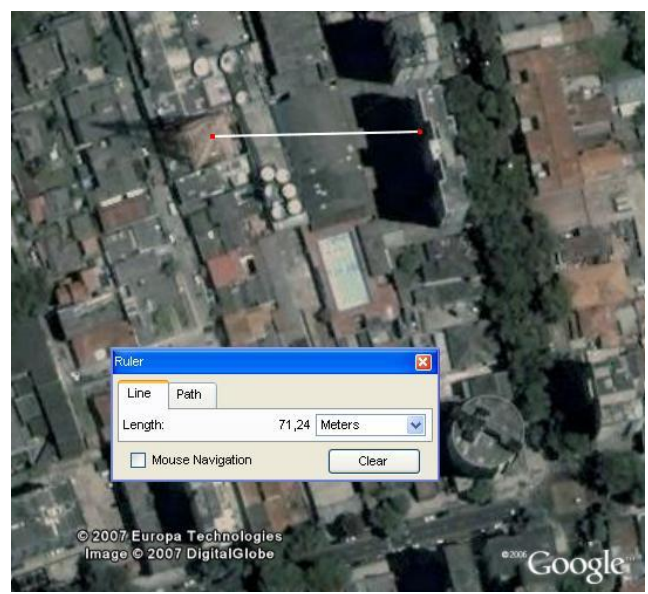

Fig. 5. Foto da antena da Liberal e o ponto de medição.

\section{TABELA 2}

Cálculo e Medição do campo elétrico do sistema irradiante de radiodifusão de sons e imagens da TV Liberal

\begin{tabular}{|c|c|}
\hline PARÁMETROS & 1 \\
\hline Potência Máxima do Transmissor $\{\mathrm{W}\}$ & 30000 \\
\hline Perdas totais no Sistema Lsistema $\{\mathrm{dB}\}$ & 2,50 \\
\hline Perdas Totais no Sistema Isistema $\{$ Linear $\}$ & 1,78 \\
\hline Ganho da Antena Gantena $\{\mathrm{dBi}\}$ & 4,68 \\
\hline Ganho da Antena Gantena $\{$ linear $\}$ & 2,94 \\
\hline $\operatorname{eirp}\{\mathrm{W}\}=$ Pxmt x Gantena / Isistema & 49559 \\
\hline DENSIDADE DE POTÊNCIA $\left\{\mathrm{W} / \mathrm{m}^{2}\right\}$ & 1,989 \\
\hline CAMPO MAGNÉTICO $\{\mathrm{A} / \mathrm{m}\}$ & 0,073 \\
\hline $\begin{array}{l}\text { CAMPO ELÉTRICO CALCULADO }\{\mathrm{V} / \mathrm{m}\} \\
\text { CAMPOELÉTRICO MEDIDO }\{\mathrm{V} / \mathrm{m}\}\end{array}$ & $\begin{array}{l}27,386 \\
24,642\end{array}$ \\
\hline
\end{tabular}

\subsection{Rádio e Televisão Marajoara LTDA}

Latitude: 01s27'43,0".

Longitude: 48s29'28,0".

Antena: situada a 131 metros.

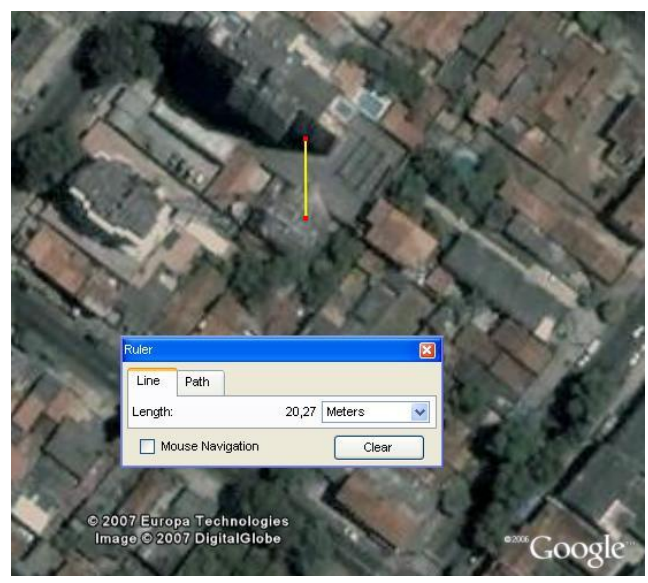

Fig. 6. Foto da antena da Record e o ponto de medição.

\section{TABELA 3}

Cálculo e Medição do campo elétrico do sistema irradiante de radiodifusão de sons e imagens da TV Record 


\begin{tabular}{|c|c|}
\hline PARÁMETROS & 1 \\
\hline Potência Máxima do Transmissor $\{\mathrm{W}\}$ & 20000 \\
\hline Perdas totais no Sistema Lsistema $\{\mathrm{dB}\}$ & 3,10 \\
\hline Perdas Totais no Sistema Isistema $\{$ Linear $\}$ & 2,04 \\
\hline Ganho da Antena Gantena $\{\mathrm{dBi}\}$ & 6,16 \\
\hline Ganho da Antena Gantena $\{$ linear $\}$ & 4,13 \\
\hline $\operatorname{eirp}\{\mathrm{W}\}=$ Pxmt x Gantena / Isistema & 40460 \\
\hline DENSIDADE DE POTÊNCIA $\left\{\mathrm{W} / \mathrm{m}^{2}\right\}$ & 20,061 \\
\hline CAMPO MAGNÉTICO $\{\mathrm{A} / \mathrm{m}\}$ & 0,231 \\
\hline $\begin{array}{l}\text { CAMPO ELÉTRICO CALCULADO }\{\mathrm{V} / \mathrm{m}\} \\
\text { CAMPO ELÉTRICO MEDIDO }\{\mathrm{V} / \mathrm{m}\}\end{array}$ & $\begin{array}{l}86,966 \\
78,264\end{array}$ \\
\hline
\end{tabular}

5.4. Rede Brasil Amazônia de Comunicação

Latitude: $01 \mathrm{~s} 25,54,0$ ".

Longitude: 48 s 28 ' 30,0 ".

Antena: situada a 116,67 metros

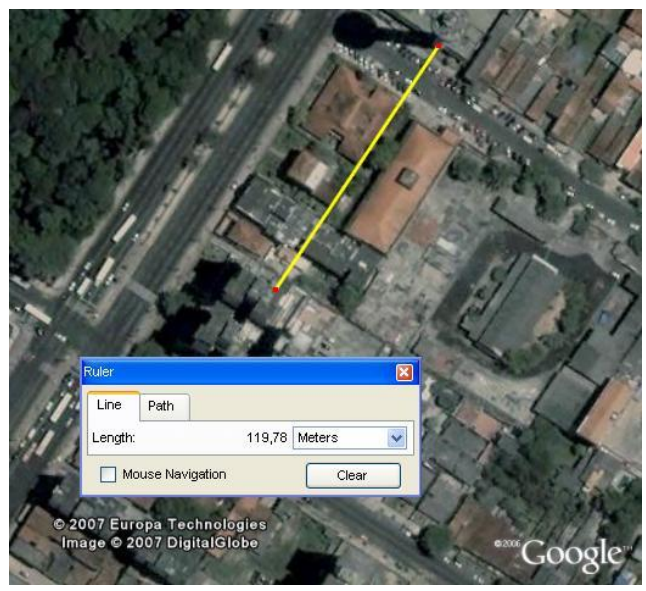

Fig. 7. Foto da antena da RBA e o ponto de medição.

\section{TABELA 4}

Cálculo e Medição do campo elétrico do sistema irradiante de radiodifusão de sons e imagens da TV RBA

\begin{tabular}{|c|c|}
\hline PARÁMETROS & 1 \\
\hline Potência Máxima do Transmissor $\{\mathrm{W}\}$ & 26000 \\
\hline Perdas totais no Sistema Lsistema $\{\mathrm{dB}\}$ & 2,60 \\
\hline Perdas Totais no Sistema Isistema $\{$ Linear $\}$ & 1,82 \\
\hline Ganho da Antena Gantena $\{\mathrm{dBi}\}$ & 6,20 \\
\hline Ganho da Antena Gantena $\{$ linear $\}$ & 4,17 \\
\hline $\operatorname{eirp}\{W\}=P x m t \times$ Gantena / Isistema & 59563 \\
\hline DENSIDADE DE POTÊNCIA $\left\{\mathrm{W} / \mathrm{m}^{2}\right\}$ & 0,846 \\
\hline CAMPO MAGNÉTICO $\{\mathrm{A} / \mathrm{m}\}$ & 0,047 \\
\hline $\begin{array}{l}\text { CAMPO ELÉTRICO CALCULADO }\{\mathrm{V} / \mathrm{m}\} \\
\text { CAMPO ELÉTRICO MEDIDO }\{\mathrm{V} / \mathrm{m}\}\end{array}$ & $\begin{array}{l}17,856 \\
16,065\end{array}$ \\
\hline
\end{tabular}

\section{CONCLUSÃO}

Este artigo teve como principal enfoque alertar os organismos reguladores e a sociedade em geral, para a necessidade de limitarmos os valores de campos elétricos, magnéticos e eletromagnéticos originados de estações transmissoras de sons e imagens, ou retirar estes transmissores de dentro dos grandes centros urbanos.

No presente estudo foram realizadas medidas dos valores de campos elétricos em edifícios próximos das estações transmissoras, com o uso de uma sonda e um analisador de espectro devidamente calibrado, e foram encontrados valores acima dos limites permitidos para exposição do corpo humano a radiação não-ionizante, originada desses transmissores.
Com isso, o presente artigo propõe um debate mais amplo a respeito do assunto, onde soluções como: a diminuição das potências dos transmissores, junto com a implantação de repetidores para continuar cobrindo a mesma região, sem a necessidade da transmissão em altas potências ou então, a retirada destes transmissores da região metropolitana das grandes cidades e implantação dos mesmos em locais seguros, sem alterar a potência de transmissão, podem ser utilizadas.

\section{REFERÊNCIAS}

[1] EL BERN A.: Radiações Não-Ionizantes: Conceitos, Riscos e Normas. Curso de Engenharia de Segurança do Trabalho, Pro Rad, p. 4-8.

[2] HOSPITAL PHYSICISTS' ASSOCIATION. Practical Aspects of Non-Ionizing Radiation Protection. Conference Report Series, 36, 1982.

[3] CRUZ, S.C.da; SIQ UEIRA, G .L.: Verificação dos níveis de radiação emitidos pelas antenas das ERBs e a percepção das comunidades próximas. Dissertação de Mestrado em Engenharia Elétrica. Pontifícia Universidade Católica do Rio de Janeiro, Departamento de Engenharia Elétrica. 2005. Cap. 4, p. 49-60.

[4] ANATEL. Regulamento sobre Limitação da Exposição a Campos Elétricos, Magnéticos e Eletromagnéticos na Faixa de Radiofrequiências entre $9 \mathrm{kHz}$ e $300 \mathrm{GHz}$. Anexo à Resolução 303 de 2 de Julho de 2002.

[5] ANATEL. SRD - Sistema de Controle de Radiodifusão: Dados das Estações de Radiodifusão de Sons e Imagens das emissoras de estudo do presente artigo. Disponível em <http://sistemasnet/SRD/CadastroTec/CaracEstacao/ Tela.asp> Acesso em 30 abr. 2007.

[6] ICNIRP (International Commission on NonIonizing Radiation Protection), "Guidelines for Limiting Exposure to Time-Varying Electric, Magnetic, and Electromagnetic Fields (up to 300Ghz)", Health Physics, Abril, 1998, Volume 74, Número 4. 